\title{
EXISTENTIAL HORN SENTENCES
}

\author{
R. C. LYNDON ${ }^{1}$
}

A. Horn [2] has shown that every sentence of a certain class, which we define precisely below, is true in a direct product of algebras whenever it is true in each component algebra. McKinsey [4] had already shown essentially that every universal sentence preserved under direct product is equivalent to one of these Horn sentences. We establish here the analogous result for existential sentences. This answers a question raised by Chang and Morel [1], who showed that the parallel result does not hold for universal-existential sentences. The problem for existential-universal sentences remains open.

An algebraic system $A$ will be taken to consist of a nonempty set of elements, upon which are defined various operations, and various relations in addition to equality. Thus $A$ is a model for a first order language $L$ with symbols for the operations and relations of $A$. (For details, see [3].) Only those models will be considered in which the equality symbol is interpreted by a relation having the usual formal properties of equality - that is, by a congruence on the algebra $A$; but we do not require that the equality symbol be interpreted by strict identity.

The direct product of "similar" algebras, that is, models for the same language, is defined in the usual way. Under this definition the direct product of an empty set of algebras is a trivial algebra, in which all relations, including equality, are universal.

A transformation of the prenex conjunctive normal form shows that every formula of $L$ is equivalent to a conditional formula, of the form

$$
\mathcal{Q} \bigwedge_{i \in I}\left[\bigwedge_{j \in J_{i}} \alpha_{\imath j} \supset \underset{k \in K_{i}}{\bigvee} \beta_{i k}\right]
$$

where $Q$ is a string of quantifiers, and the $\alpha_{i j}$ and $\beta_{i k}$ are atomic formulas indexed by finite sets $I, J_{i}, K_{i}$. A conditional formula is a Horn formula if each $K_{i}$ has at most one element; for $K_{i}$ empty, the expression in brackets is interpreted as $\sim \bigwedge_{j \in J_{i}} \alpha_{i j}$. It is a strict Horn formula if each $K_{i}$ has exactly one element.

Lemma. If a Horn formula $H$ without quantifiers implies a disjunc-

Received by the editors February 7, 1959.

1 The author is associated with a National Science Foundation project, Grant G-2371. 
tion $\gamma_{1} \vee \cdots \vee \gamma_{n}$ of atomic formulas, where $n>0$, then $H$ implies $\gamma_{\nu}$ for some $\nu=1,2, \cdots, n$.

The formula $H$, together with the axioms for equality, is equivalent to a set $H^{*}$ of conditions h, each of one of the two forms

$$
\begin{aligned}
& \mathrm{h}_{1}: \alpha_{1} \wedge \cdots \wedge \alpha_{m} \supset \beta, \\
& \mathrm{h}_{2}: \sim\left(\alpha_{1} \wedge \cdots \wedge \alpha_{m}\right)
\end{aligned}
$$

where $\alpha_{1}, \cdots, \alpha_{m}$ and $\beta$ are atomic formulas, and $m \geqq 0$. If $F$ is any set of atomic formulas of $L$, let $F^{*}$ be the set consisting of all $\alpha$ in $F$, together with $\sim \alpha$ for all atomic formulas $\alpha$ not in $F$. If $F^{*}$ is consistent with $H^{*}$, it is clear that $F$ satisfies the conditions

$\mathrm{h}_{1}^{\prime}:$ if $\alpha_{1}, \cdots, \alpha_{m-1}$, and $\alpha_{m}$ are in $F$, then $\beta$ is in $F$,

$\mathrm{h}_{2}^{\prime}:$ not all of $\alpha_{1}, \cdots, \alpha_{m-1}$, and $\alpha_{m}$ are in $F$, corresponding to each $\mathrm{h}$ in $H^{*}$. Conversely, for any $F, F^{*}$ is consistent (in predicate calculus without axioms for equality), and if $F$ satisfies the conditions $\left(\mathrm{h}_{1}^{\prime}, \mathrm{h}_{2}^{\prime}\right), F^{*}$ implies $\mathrm{h}$ for each $\mathrm{h}$ in $H^{*}$, whence $F^{*}$ is consistent with $H^{*}$.

From the form of the conditions $\left(\mathrm{h}_{1}^{\prime}, \mathrm{h}_{2}^{\prime}\right)$ it is clear that, for $n>0$, if $F_{1}, \cdots, F_{n}$ satisfy these conditions, then $F=F_{1} \cap \cdots \cap F_{n}$ also satisfies them. Thus, if each of $F_{1}^{*}, \cdots, F_{n}^{*}$ is consistent with $H^{*}$, so also is $F^{*}$. To prove the lemma we show that, if each of $\sim \gamma_{1}, \cdots$, $\sim \gamma_{n}$ is consistent with $H^{*}$, then $\left\{\sim \gamma_{1}, \cdots, \sim \gamma_{n}\right\}$ is consistent with $H^{*}$. That $\sim \gamma_{\nu}$ is consistent with $H^{*}$ implies the existence of a function assigning the truth values 0 and 1 to atomic formulas of $L$ under which $\gamma_{\nu}$ receives the value 0 , while each formula $h$ of $H^{*}$ acquires the value 1 . If $F_{\nu}$ is the set of atomic formulas receiving the value 1 under this assignment, then $F_{\nu}^{*}$ is consistent with $H^{*}$, and $F_{\nu}$ does not contain $\gamma_{\nu}$. But then $F^{*}$ is consistent with $H^{*}$, for $F=F_{1} \cap \cdots \cap F_{n}$, and $F$ contains none of $\gamma_{1}, \cdots, \gamma_{n}$. It follows that $\left\{\sim \gamma_{1}, \cdots, \sim \gamma_{n}\right\}$ is consistent with $H^{*}$, as required.

Proposition 1. Let $S$ and $T$ be existential sentences of a language $L$ such that $T$ holds in every direct product of a (possibly empty) set of algebras satisfying $S$. Then there exists an existential strict Horn sentence $U$ such that $S$ implies $U$ and $U$ implies $T$.

We note that the converse follows immediately from Horn's result.

To prove the proposition, we may first replace $L$ by a language containing, in addition to the equality symbol, only the finite number of operation and relation symbols that occur in $S$ and $T$. Let $Z(z)$ be the conjunction of formulas $z=\omega(z, z, \cdots, z)$ for each operation symbol $\omega$, and $\rho(z, z, \cdots, z)$ for each relation symbol $\rho$. Then the sentence $Z=\exists z \cdot Z(z)$ expresses of an algebra that it contains a trivial 
subalgebra. Suppose that $A$ is a direct product of algebras satisfying $S^{\prime}=S \vee Z$. Then $A$ contains a subalgebra $B$ that is a direct product of algebras satisfying $S$ together with trivial algebras. Since $B$ is isomorphic to the direct product of its nontrivial components, which satisfy $S, B$ satisfies $T$. Since $T$ is existential, and holds for the subalgebra $B$ of $A, T$ holds for $A$. Thus the hypothesis of the proposition holds with $S$ replaced by $S^{\prime}$. Since $S$ implies $S^{\prime}$, the conclusion for $S^{\prime}$ would imply the conclusion for $S$. Thus, in the remainder of the argument, it suffices to consider $S^{\prime}$ in place of $S$; dropping primes, we may henceforth assume that $Z$ implies $S$, that is, that $S$ holds for every algebra that contains a trivial subalgebra.

Supposing $S$ written in conditional form, as above, it now follows that none of the $K_{i}$ are empty. Replace each part $\Lambda_{j \in J_{i}} \alpha_{i j} \supset \bigvee_{k \in K_{i}} \beta_{i k}$ by $\bigvee_{k \in K_{i}}\left[\Lambda_{j \in J_{i}} \alpha_{i j} \supset \beta_{i k}\right]$, and distribute the conjunction with respect to $i \in I$, and the existential quantifiers, over the disjunctions. This shows that $S$ is equivalent to a disjunction of existential strict Horn sentences

$$
\exists x_{1} \cdots x_{P} \wedge\left[\bigwedge_{i \in I} \alpha_{j \in J_{i}} \supset \beta_{i \kappa(i)}\right]
$$

for all elements $\kappa=\langle\kappa(i)\rangle$ in the cartesian product $\mathrm{II}_{i \in I} K_{i}$.

We may now suppose that $S=H^{1} \vee \cdots \vee H^{N}$, where $H^{n}$ $=\exists x_{1} \cdots x_{P} \bar{H}^{n}(x)$, and $\bar{H}^{n}(x)$ stands for a strict Horn formula $\bar{H}^{n}\left(x_{1}, \cdots, x_{P}\right)$ without quantifiers. We may suppose also that $T=\exists y_{1} \cdots y_{R} \bar{T}(y)$ where $\bar{T}(y)$ stands for a conditional formula $\bar{T}\left(y_{1}, \cdots, y_{R}\right)$ without quantifiers.

Let $A$ be the direct product of algebras $A^{1}, \cdots, A^{N}$, and, for each $n$, let $a_{1}^{n}, a_{2}^{n}, \cdots, a_{P}^{n}$ be elements of $A^{n}$. If $\bar{H}^{n}\left(a^{n}\right)=\bar{H}^{n}\left(a_{1}^{n}, \cdots, a_{P}^{n}\right)$ holds for each $n$, then each $A^{n}$ satisfies $H^{n}$ and so $S$, whence $A$ must satisfy $T$. Therefore $H(a)=\bar{H}^{1}\left(a^{1}\right) \wedge \cdots \wedge \bar{H}^{N}\left(a^{N}\right)$ is inconsistent with the failure of $T$ on $A$, hence with the truth of $\forall y_{1} \cdots y_{R}$ . $\sim \bar{T}(y)$ on $A$, and thus with some finite set of instances $\sim \bar{T}(b(1))$, $\cdots, \sim \bar{T}(b(M))$ of $\sim \bar{T}(y)$, obtained by replacing each $y_{r}$ by an element $b_{r}(m)$ of $A$. Here in fact each $b_{r}(m)=\left\langle b_{r}^{1}(m), \cdots, b_{r}^{N}(m)\right\rangle$, a vector whose component $b_{r}^{n}(m)$ is some expression built out of $a_{1}^{n}, \cdots, a_{P}^{n}$ by means of the operation symbols $\omega$. Replace $T$ by the conditional form of the equivalent sentence

$$
\begin{aligned}
& \exists y_{1}(1), \cdots, y_{R}(1), \cdots, y_{1}(M), \cdots, y_{R}(M) \\
& \qquad \bar{T}(y(1)) \vee \cdots \vee \bar{T}(y(M)) .
\end{aligned}
$$

Then we may suppose that, reverting to the earlier notation, $H(a)$ implies a single instance $\bar{T}(b)$ of $\bar{T}(y)$. Here the components of the 
$b_{r}$ are still expressions $t\left(a^{n}\right)$ in the $a_{1}^{n}, \cdots, a_{P}^{n}$. Adjoining to the $\bar{H}^{n}(x)$ suitable new clauses of the form $x^{\prime}=t\left(x_{1}, \cdots, x_{P}\right)$ and prefixing new existential quantifiers, we obtain, upon reverting to the original notation, that each component $b_{r}^{n}$ of a $b_{r}$ is one of $a_{1}^{n}, \cdots, a_{P}^{n}$.

To restate the result of these considerations, if $f$ is any function from $\{1,2, \cdots, N\}$ into $\{1,2, \cdots, P\}$, let $a_{f}$ denote the element $\left\langle a_{f(1)}^{1}, \cdots, a_{f(N)}^{N}\right\rangle$ of $A$. Then we have justified the supposition that for some elements $a_{f_{1}}, \cdots, a_{f_{R}}$ of $A, H(a)$ implies $\bar{T}\left(a_{f}\right)$ $=\bar{T}\left(a_{f_{1}}, \cdots, a_{f_{R}}\right)$.

If $\gamma\left(a_{f}\right)=\gamma\left(a_{f_{1}}, \cdots, a_{f_{R}}\right)$ is an atomic formula (in $T$ ), write $\gamma\left(a_{f}\right)^{n}=\gamma\left(a_{f_{1}(n)}^{n}, \cdots, \quad a_{f_{R}(n)}^{n}\right)$, and $\gamma\left(a_{f}\right)^{*}=\gamma\left(a_{f}\right)^{1} \wedge \cdots \wedge \gamma\left(a_{f}\right)^{N}$. The definition of the direct product takes $\gamma\left(a_{f}\right)$ as equivalent to $\gamma\left(a_{f}\right)^{*}$, and thus interprets $\bar{T}\left(a_{f}\right)$ as meaning $\bar{T}\left(a_{f}\right)^{*}$, the result of replacing each $\gamma\left(a_{f}\right)$ in $T\left(a_{f}\right)$ by $\gamma\left(a_{f}\right)^{*}$. We have then that $H(a)$ implies $\bar{T}\left(a_{f}\right)^{*}$, purely formally within the language $L$ (with equality).

The conditional formula $\bar{T}(y)$ will have the form $\bar{T}(y)=\bigwedge_{i \in \mathrm{I}} C_{i}(y)$, where each $C_{i}(y)=A_{i}(y) \supset B_{i}(y), \quad A_{i}(y)=\bigwedge_{j \in J_{i}} \alpha_{i j}(y), \quad B_{i}(y)$ $=\mathrm{V}_{k \in K_{i}} \beta_{i k}(y)$. Since $T$ holds on a trivial algebra, no $K_{i}$ is empty. For each $i$, since $H(a)$ implies $T\left(a_{f}\right)^{*}$ it implies $C_{i}\left(a_{f}\right)^{*}$, whence $H_{i}$ $=H(a) \wedge A_{i}\left(a_{f}\right)^{*}$ implies $B_{i}\left(a_{f}\right)^{*}$. But

$$
B_{i}\left(a_{f}\right)^{*}=\underset{k \in K_{i}}{\vee} \bigwedge_{n=1}^{N} \beta_{i k}\left(a_{f}\right)^{n} \equiv \bigwedge_{\phi \in N^{K}{ }_{i}} \underset{k \in K_{i}}{\vee} \beta_{i k}\left(a_{f}\right)^{\phi(k)},
$$

whence $H_{i}$ implies $\bigvee_{k \in K_{i}} \beta_{i k}\left(a_{f}\right)^{\phi(k)}$ for every $\phi$ in $N^{K_{i}}$.

For $i$ in $I$ still fixed, suppose that for no $k$ in $K_{i}$ does $H_{i}$ imply $\beta_{i k}\left(a_{f}\right)^{*}$. Then, for each $k$ in $K_{i}$ there exists $\phi(k)$ in $N$ such that $H_{i}$ does not imply $\beta_{i k}\left(a_{f}\right)^{\phi(k)}$. Since it was established that $H_{i}$ implies $\bigvee_{k \in K} \beta_{i k}\left(a_{f}\right)^{\phi(k)}$, and $H_{i}$ is a Horn formula without quantifiers, this contradicts the lemma.

We conclude that, for each $i$ in $I$, there exists $k_{i}$ in $K_{i}$ such that $H_{i}$ implies $\beta_{i k_{i}}\left(a_{f}\right)^{*}$. Construct $U$ from $T$ by replacing each disjunction $\bigvee_{k \in K_{i}} \beta_{i k}(y)$ by the single atomic formula $\beta_{i k_{i}}(y)$. It is immediate that $U$ is an existential strict Horn sentence, and that $U$ implies $T$.

It remains to show that $S$ implies $U$, that is, that each $H^{n}$ implies $U$. Fixing $n$, take $A^{n^{\prime}}$ trivial for all $n^{\prime} \neq n$. Then each $A^{n^{\prime}}$ satisfies the strict Horn sentence $H^{n^{\prime}}$, and $A$ satisfies $U$ provided $A^{n}$ satisfies $H^{n}$. Since $A$ is isomorphic to $A^{n}, A^{n}$ satisfies $U$ provided it satisfies $H^{n}$ : that is, $H^{n}$ implies $U$.

Proposition 2. Let $S$ and $T$ be existential sentences of a language $L$ such that $T$ holds in every direct product of a nonempty set of algebras 
satisfying $S$. Then there exists an existential Horn sentence $U$ such that $S$ implies $U$ and $U$ implies $T$.

If $S$ holds for a trivial algebra, $T$ must also, and the assertion is contained in Proposition 1. Assuming then that $S$ fails for trivial algebras, we indicate those modifications of the previous argument necessary to prove the present proposition.

Let $W(x)=\bar{Z}\left(x_{1}\right) \wedge x_{1}=x_{2} \wedge \cdots \wedge x_{1}=x_{P}$; then $W\left(a^{n}\right)$ asserts that the subalgebra $A_{0}^{n}$ of $A^{n}$ generated by $a_{1}^{n}, \cdots, a_{P}^{n}$ is trivial. Thus $H_{0}^{n}=\bar{H}^{n}\left(a^{n}\right) \bigvee W\left(a^{n}\right)$ implies that $A_{0}^{n}$ either satisfies $S$ or is trivial. Since $S$, and so each $H^{n}$, fails on a trivial algebra, $H^{n}\left(a^{n}\right)$ implies $\sim W\left(a^{n}\right)$. The formula $H_{0}=H_{0}^{1} \wedge \cdots \wedge H_{0}^{N}$ implies that each $A_{0}^{n}$ satisfies $S$ or is trivial. The formula $Y=\sim\left(W\left(a^{1}\right) \wedge \cdots \wedge W\left(a^{N}\right)\right)$ asserts that not all $A_{0}^{n}$ are trivial.

$\mathrm{H}_{0} \wedge Y$ is a Horn formula without quantifiers. It implies that the direct product $A_{0}$ of the $A_{0}^{n}$ is a direct product of factors that either satisfy $S$ or are trivial, and that not all factors are trivial. Hence $A_{0}$ is isomorphic to a direct product of a nonempty set of algebras satisfying $S$, and therefore satisfies $T$. It follows that $A$ satisfies $T$. We have then that $H_{0} \vee Y$ implies that $A$ satisfies $T$, and we obtain a Horn sentence $U$ exactly as before, except that we refrain from attempting to choose $k_{i}$ from those $K_{i}$ which may, now, be empty. Then $U$ implies $T$ as before, and the same device as before shows that each $H^{n}$ implies $U$, in view of the fact that $\bar{H}^{n}\left(a^{n}\right)$ implies $\sim W\left(a^{n}\right)$ and hence implies $Y$.

\section{BIBLIOGRAPHY}

1. C. C. Chang and Anne C. Morel, On closure under direct product, J. Symb. Logic vol. 23 (1958) pp. 149-154.

2. A. Horn, On sentences which are true of direct unions of algebras, J. Symb. Logic vol. 16 (1951) pp. 14-21.

3. Roger C. Lyndon, An interpolation theorem in the predicate calculus, Pacific J. Math. vol. 9 (1959) pp. 129-142.

4. J. C. C. McKinsey, The decision problem for some classes of sentences without quantifiers, J. Symb. Logic vol. 8 (1943) pp. 61-76.

UNIVERSITY OF Michigan 